\title{
Importancia de la acreditación de laboratorios de organismos de regulación enológicos
}

\section{Importance of the laboratories' accreditation of oenological regulation organizations}

\author{
G. Gil, M. Cedrés, D. Herrera, J. Balado, y F. Capello \\ INAVI, Departamento de Laboratorio, Dr. Pouey 463, Las Piedras, Uruguay
}

\begin{abstract}
Resumen. A medida que aumenta la globalización de los suministros de alimentos, resulta cada vez más evidente la necesidad de reforzar los sistemas que velan por la inocuidad de los alimentos en todos los países. La acreditación es el reconocimiento formal, que hace una tercera parte, de que un organismo cumple con los requisitos especificados y es competente para desarrollar tareas específicas de evaluación de la conformidad. INAVI como organismo regulador de la vitivinicultura de la República Oriental del Uruguay cuenta desde el año 2011 con un Laboratorio Acreditado por el OUA (Organismo Uruguayo de Acreditación) en la Norma ISO/IEC 17025:2005. En la actualidad cuenta con 16 alcances en dos matrices diferentes vinos y sidras asegurando así su competencia técnica. Los Organismos Reguladores en Enología con frecuencia deben tomar decisiones en referencia a la salud y bienestar de los consumidores, protección del medio ambiente, control del cumplimiento de requisitos existentes reglamentarios y legales, generación de nuevos requisitos y regulaciones, así como asignación de recursos técnicos y financieros. Para poder tomar estas decisiones los Organismos de Regulación deben tener confianza en los resultados generados, la acreditación de sus laboratorios de ensayo genera la confianza necesaria para el desarrollo de dichas actividades.
\end{abstract}

\begin{abstract}
As the globalization of food supplies increases, the need to strengthen systems that ensure the safety of food in all countries becomes increasingly evident. Accreditation is the formal recognition, made by a third part, that an organism meets the specified requirements and is competent to perform specific tasks of conformity assessment. INAVI, as the regulator of national vitiviniculture, has a Laboratory Accredited by the OUA (Uruguayan Accreditation Organization) in the ISO/IEC 17025:2005 Standard since 2011. Currently, it has 16 scopes in two matrices different wines and ciders thus ensuring their technical competence. Regulatory Organizations in Oenology often has to make decisions regarding the health and wellness of consumers, protection of the environment, control of compliance with existing regulatory and legal requirements, generation of new requirements and regulations, as well as allocation of technical and financial resources. In order to make these decisions, the Regulatory Organizations must have confidence in the results generated, the accreditation of their testing laboratories generates the confidence necessary for the development of this activities.
\end{abstract}

A medida que aumenta la globalización de los suministros de alimentos, resulta cada vez más evidente la necesidad de reforzar los sistemas que velan por la inocuidad de los alimentos en todos los países.

Los cambios en la demanda de los consumidores, los avances tecnológicos aplicados en la industria, así como las exigencias de los mercados internacionales y los requisitos en seguridad alimentaria, requieren estudio y actualización permanente de los sistemas nacionales de control de los alimentos, siendo los organismos reguladores los responsables de esta función.

El Instituto Nacional de Vitivinicultura (I.NA.VI.) fue creado en Uruguay por la Ley 15.903 en 1987, en él se encuentran representados todos los segmentos productivos del sector vitivinícola. Entre sus funciones, el Instituto debe asesorar al Poder Ejecutivo y fiscalizar el cumplimiento de las normas legales vigentes que se dicten en materia vitivinícola. Asimismo, debe promover la vitivinicultura a través de la investigación, extensión y divulgación.

Cumpliendo con este cometido, el Departamento de Laboratorio del I.NA.VI. realiza actividades que se relacionan con la fiscalización y control bromatológico de los productos de origen vitivinícola mediante métodos reconocidos y validados por la normativa internacional.

La acreditación es el reconocimiento formal, que hace una tercera parte, de que un organismo cumple con los requisitos especificados y es competente para desarrollar tareas específicas de evaluación de la conformidad.

El presente trabajo tiene como objetivo remarcar y valorizar la importancia que la Acreditación tiene en los Organismos Reguladores, otorgándoles las siguientes 
Tabla 1. Alcance de Acreditación [2,3].

\begin{tabular}{|c|c|c|c|}
\hline Matriz & Ensayo & Rango & Método \\
\hline \multirow{8}{*}{$\begin{array}{c}\text { Vinos } \\
\text { y } \\
\text { Sidras }\end{array}$} & $\begin{array}{l}\text { Relación } \\
\text { isotópica } \\
\text { de }{ }^{13} \mathrm{C} /{ }^{12} \mathrm{C} \\
\text { de etanol }\end{array}$ & $\begin{array}{l}\delta^{13} \mathrm{C}= \\
-32 \% \\
\mathrm{a}-11 \%\end{array}$ & $\begin{array}{l}\text { Resolución } \\
\text { OIV/OENO 17-2001. } \\
\text { Método OIV-MA-AS } \\
\text { 312-06: R 2009. } \\
\text { Determinación por } \\
\text { espectrometría de } \\
\text { masa isotópica de la } \\
\text { relación de isótopos } \\
{ }^{13} \mathrm{C} /{ }^{12} \mathrm{C} \text { del etanol del } \\
\text { vino. }\end{array}$ \\
\hline & $\begin{array}{l}\text { Relación } \\
\text { isotópica } \\
\mathrm{de}^{18} \mathrm{O} /{ }^{16} \mathrm{O} \\
\text { del agua }\end{array}$ & $\begin{array}{l}\delta^{18} \mathrm{O}= \\
-7 \% \text { a } 4 \% 0\end{array}$ & $\begin{array}{l}\text { Resolución } \\
\text { OIV/OENO 353/2009. } \\
\text { Método OIV-MA-AS } \\
\text { 2-12: R 2009. } \\
\text { Método de } \\
\text { determinación de la } \\
\text { relación de isótopos } \\
{ }^{18} \mathrm{O} /{ }^{16} \mathrm{O} \text { del agua en } \\
\text { vinos y mostos. }\end{array}$ \\
\hline & $\begin{array}{l}\text { Grado } \\
\text { alcohólico } \\
\text { (Alcohol\% } \\
\text { vol.) }\end{array}$ & 4 a $18 \%$ vol. & $\begin{array}{l}\text { Resolución } \\
\text { OIV/OENO } \\
\text { 566/2016-Método } \\
\text { OIV-MA-AS 312-01 } \\
\text { A: R 2016.4 B } \\
\text { Medición del grado } \\
\text { alcohólico del vino por } \\
\text { densimetría } \\
\text { electrónica utilizando } \\
\text { un resonador de } \\
\text { flexión }\end{array}$ \\
\hline & $\begin{array}{l}\text { Masa } \\
\text { volúmica } \\
\text { (Dens) }\end{array}$ & $\begin{array}{l}0.98460 \mathrm{a} \\
1.02940 \mathrm{~g} / \mathrm{mL}\end{array}$ & $\begin{array}{l}\text { Resolución } \\
\text { OIV/OENO 437- } \\
\text { 2012- Método OIV- } \\
\text { MA-AS 2- 01 A: } \\
\text { R } 2012 \\
\text { Masa volúmica a } 20{ }^{\circ} \mathrm{C} \\
\text { y densidad relativa } \\
\text { a } 20^{\circ} \mathrm{C} \text { medida } \\
\text { por densimetría } \\
\text { electrónica mediante } \\
\text { resonador de flexión. }\end{array}$ \\
\hline & $\begin{array}{l}\text { Acidez } \\
\text { volátil }\end{array}$ & $\begin{array}{l}0.1 \mathrm{a} \quad 2.0 \mathrm{~g} \\
\mathrm{H}_{2} \mathrm{SO}_{4} / \mathrm{L}\end{array}$ & $\begin{array}{l}\text { Resolución } \\
\text { OIV/OENO 549/2015 } \\
\text { - Método OIV-MA- } \\
\text { AS 313-02 : } \\
\text { R } 2015 \text { Acidez Volátil. }\end{array}$ \\
\hline & $\begin{array}{l}\text { Anhídrido } \\
\text { sulfuroso } \\
\text { total }\end{array}$ & $\begin{array}{l}15 \mathrm{a} \\
480 \mathrm{mg} / \mathrm{L}\end{array}$ & $\begin{array}{l}\text { Resolución } \\
\text { OIV/OENO 377/2009. } \\
\text { Método OIV-MA-AS } \\
\text { 323-04A: } \\
\text { R 2012 Anhídrido } \\
\text { sulfuroso. }\end{array}$ \\
\hline & $\begin{array}{l}\text { Azúcares } \\
\text { reductores }\end{array}$ & $\begin{array}{l}1.0 \mathrm{a} \\
250.0 \mathrm{~g} / \mathrm{L}\end{array}$ & $\begin{array}{l}\text { PNL 21, versión } 3 \\
\text { Basado en Norma } \\
\text { UNIT 615-80. } \\
\text { Determinación del } \\
\text { contenido de azúcares } \\
\text { reductores }\end{array}$ \\
\hline & Metanol & $\begin{array}{l}50 \mathrm{a} \\
500 \mathrm{mg} / \mathrm{L}\end{array}$ & $\begin{array}{l}\text { PNL 24, versión 2, } \\
\text { basado en Resolución } \\
\text { OIV/OENO 480/2014 } \\
\text { - Método OIV-MA- } \\
\text { AS312-03 A: R2015. } \\
\text { Metanol }\end{array}$ \\
\hline
\end{tabular}

Gráfico Alcohol Muestra Control 2018

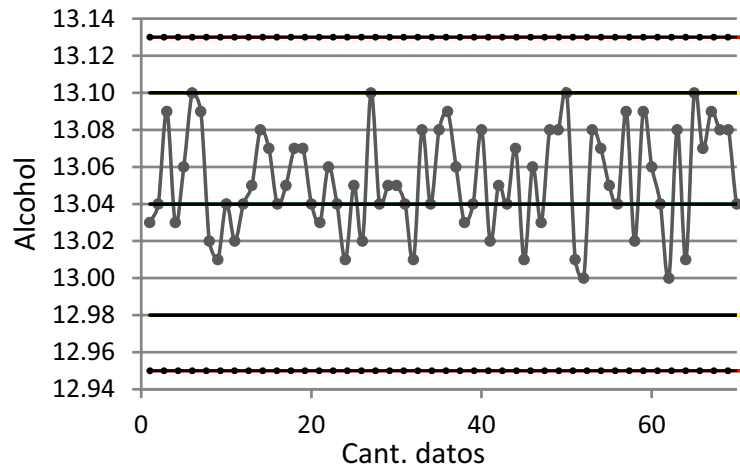

Figura 1. Gráfico de Control para Alcohol en vino.

ventajas: confiabilidad, uniformidad y reconocimiento, lo que permite facilitar el comercio internacional y la apertura de nuevos mercados, entre otros.

INAVI como organismo regulador de la vitivinicultura de la República Oriental del Uruguay, cuenta desde el año 2011 con un Laboratorio Acreditado por el OUA (Organismo Uruguayo de Acreditación) en la Norma ISO/IEC 17025:2005 [1]. En la actualidad el alcance es de 16 ensayos en dos matrices diferentes vinos y sidras que se detallan Tabla 1. Alcance de Acreditación con sus correspondientes rangos.

El ciclo de mejora continua se implementa con un robusto sistema de aseguramiento de la calidad que comprende un conjunto de acciones, planificadas y sistematizadas llevadas a cabo por el Laboratorio y que son necesarias para proporcionar la confianza adecuada de que los resultados analíticos ofrecidos satisfacen los requisitos establecidos, según la Política de Calidad del Laboratorio.

Estas acciones son llevadas a cabo mediante:

i. - El uso de material de referencia interno en cada serie analítica. Por ejemplo en la Fig. 1. se muestra un gráfico de control para el análisis de Alcohol realizado con material de referencia interno diario por serie.

ii. - La participación desde el 2009 con frecuencia mensual en ensayos de aptitud cuyos beneficios para el Laboratorio [4] son:

- Evaluar la precisión de los resultados analíticos.

- Disponer de evidencia independiente de desempeño.

- El aumento de la confiabilidad de sus resultados y de la calidad de su trabajo técnico debido al reconocimiento externo e internacional.

- Controlar y mejorar el rendimiento de sus análisis a corto, mediano y largo plazo.

- La reducción de incertidumbres asociadas con decisiones que afecten la protección de la salud humana y el medio ambiente.

La Tabla 2 muestra el resultado correspondiente al mes de Mayo 2018 del Ensayo de Aptitud para diferentes ensayos, el promedio de los participantes para cada parámetro y el parámetro de desempeño $\mathrm{z}$-score que permite la interpretación y comparación del resultado emitido por el Laboratorio considerándose como 
Tabla 2. Resultado de Ensayo de Aptitud correspondiente al mes de Mayo 2018.

\begin{tabular}{|c|c|c|c|c|}
\multicolumn{1}{c|}{} & $\mathrm{X}$ & $\mathrm{VT}$ & Valor emitido & $\mathrm{Z}$ \\
\hline Alcohol $(\% \mathrm{vol})$ & 12.23 & 0.19 & 12.22 & -0.11 \\
\hline $\begin{array}{c}\text { Densidad } \\
(\mathrm{g} / \mathrm{mL})\end{array}$ & 0.98899 & 0.00030 & 0.98893 & -0.40 \\
\hline $\begin{array}{c}\mathrm{SO}_{2} \text { Total } \\
(\mathrm{mg} / \mathrm{L})\end{array}$ & 110 & 21 & 108 & -0.19 \\
\hline $\begin{array}{c}\text { Acidez Volátil } \\
(\mathrm{meq} / \mathrm{L})\end{array}$ & 4.6 & 1.7 & 5.3 & 0.82 \\
\hline $\begin{array}{c}\text { Az. Reductores } \\
(\mathrm{g} / \mathrm{L})\end{array}$ & 2.1 & 0.8 & 2.2 & 0.25 \\
\hline Metanol (mg/L) & 26 & 15 & 25 & -0.13 \\
\hline
\end{tabular}

Alcohol Setiembre 2017- Julio 2018

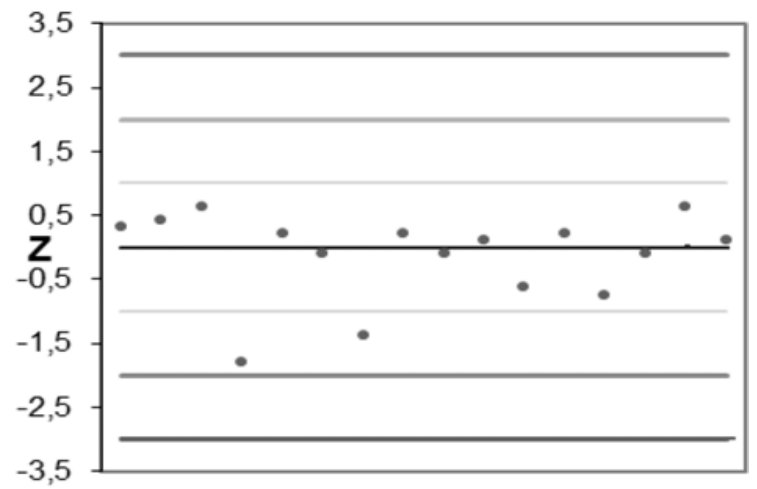

Figura 2. Resultados de z-score de una campaña anual interlaboratorial para Alcohol en vino.

satisfactorio un $\mathrm{z}$ score menor o igual a 2 en valor absoluto.

En la Fig. 2. Se muestra diferentes z-score obtenidos en la campaña Setiembre 2017 - Julio 2018 interlaboratorial para Alcohol en vino.

iii. Uso de materiales de referencia externos que aseguran la calidad de la medición, son utilizados en la validación de los métodos, calibración y evaluación de la incertidumbre [5].

iv. Utilización de métodos reconocidos, validados y documentados.

v. Personal técnico competente y comprometido.

vi. Equipos calificados, aptos con un plan de mantenimiento y calibración.

vii. Compromiso del equipo de trabajo en el análisis de riesgos [6], detección de no conformidades y seguimiento de acciones correctivas.

viii. Repeticiones de análisis (no ciego, ciego) con una frecuencia mínima anual, debiéndose obtener valores que no difieran más de la reproducibilidad del método.

ix. Diseño de documentación eficiente aplicables al Laboratorio.
Los resultados de estas acciones brindan al Laboratorio:

- Control estadístico del proceso de análisis.

- Detección de errores sistemáticos.

- Definición de acciones correctivas.

- Evaluación de la calidad analítica.

- Verificación de la capacidad del Laboratorio para cumplir requisitos normalizados.

- Trazabilidad [7] metrológica a patrones internacionales.

- Robustez de valores asignados.

- Reconocimiento formal de la competencia técnica mediante un proceso de Auditorías muy completo.

- Reconocimiento internacional de los resultados a través de un sistema de acuerdos internacionales.

- Evaluación continua del Laboratorio por el Organismo de Acreditación.

- Ser un elemento de réplica de calidad en el Sector Vitivinícola.

Mantener la Acreditación del Departamento de Laboratorio del I.NA.VI. y aumentar su alcance es un desafío continuo que se logra con un sólido trabajo en equipo y el compromiso de todos los miembros del Departamento de Laboratorio.

Los Organismos Reguladores en Enología con frecuencia deben tomar decisiones referentes a la salud y bienestar de los consumidores, protección del medio ambiente, control del cumplimiento de los requisitos existentes reglamentarios y legales vigentes, generación de nuevos requisitos y regulaciones, así como asignación de recursos técnicos y financieros. Para poder tomar estas decisiones los Organismos de Regulación deben tener confianza en los resultados generados, la acreditación de sus laboratorios de ensayo genera la confianza necesaria para el desarrollo de dichas actividades.

\section{Referencias}

[1] UNIT-ISO/IEC 17025.2005, Requisitos Generales Para la Competencia de los Laboratorios de Ensayo y de Calibración (2005)

[2] OIV, Compendio de los métodos Internacionales de análisis de los Vinos y de los Mostos 1, 2 (2018)

[3] UNIT Norma 615-80 Método de Hagedorn - Hensen (1980)

[4] OUA, Política y Requisitos Para la Participación en Ensayos de Aptitud/Comparaciones Interlaboratorios/controles externos (2017)

[5] ISO/IEC JCMG Guide to the Expression of Uncertainty in Measurement (GUM) 100:2008 Geneva (2008)

[6] UNIT-ISO/IEC 17025:2017, Requisitos Generales Para la Competencia de los Laboratorios de Ensayo y de Calibración (2017)

[7] OUA OUADOC016 Trazabilidad (2015) 